Canadian University Music Review

Revue de musique des universités canadiennes

\title{
Poétiques et politiques du style Kaluli
}

\section{Steven Feld}

Volume 10, numéro 2, 1990

Alternative Musicologies

Les Musicologies Alternatives

URI : https://id.erudit.org/iderudit/1014888ar

DOI : https://doi.org/10.7202/1014888ar

Aller au sommaire du numéro

\section{Éditeur(s)}

Canadian University Music Society / Société de musique des universités canadiennes

\section{ISSN}

0710-0353 (imprimé)

2291-2436 (numérique)

Découvrir la revue

\section{Citer cet article}

Feld, S. (1990). Poétiques et politiques du style Kaluli. Canadian University Music Review / Revue de musique des universités canadiennes, 10(2), 103-136. https://doi.org/10.7202/1014888ar

All Rights Reserved @ Canadian University Music Society / Société de musique des universités canadiennes, 1990
Ce document est protégé par la loi sur le droit d’auteur. L’utilisation des services d'Érudit (y compris la reproduction) est assujettie à sa politique d'utilisation que vous pouvez consulter en ligne.

https://apropos.erudit.org/fr/usagers/politique-dutilisation/ 


\section{POÉTIQUES ET POLITIQUES DU STYLE KALULI}

\section{Steven Feld}

. . . la musique est reliée à la société de la même manière que la conscience: la société se retrouve créativement 'dans' chaque pièce de musique et est, de ce fait, articulée par elle.

John Shepherd

L'obstination à opposer la vie et l'art est liée à l'expérience d'un monde étranger. Et l'échec à vouloir reconnaître la portée universelle et la dignité ontologique du jeu conduit à une abstraction qui nous empêche de voir l'interdépendance de l'une et de l'autre.

Hans-Georg Gadamer

Ma première préoccupation face à une « musicologie alternative » est que l'on insiste sur l'emploi de cette expression au pluriel. À mon avis, les musicologies alternatives doivent comprendre les discours sur la musique qui, dans le langage érudit et dominant de la musicologie, sont laissés de côté ou considérés comme tabous. Qu'elles se fondent sur une résistance au dit langage ou sur des manières autres d'imaginer le sujet, les musicologies alternatives doivent présenter des critiques de ce langage dominant; plus spécifiquement doivent-elles discuter la manière dont ce langage reproduit systématiquement une perspective de «la musique» et une érudition musicale normatives et eurocentriques. Le combat que doivent mener les musicologies alternatives est multiple; il leur faut en effet lutter en faveur de la découverte et de la reconnaissance de réalitiés musicales diverses, dénoncer la formation de faux consensus dans l'érudition musicale, dénoncer aussi le fait que la musicologie $\mathrm{a}$, de manière perverse, restreint son champ d'étude à quatre siècles d'activité musicale occidentale européenne plutôt que de l'ouvrir à l'hétérogénéité musicale humaine de deux millions d'années.

Dans un pareil contexte, il me paraît nécessaire de rappeler la parenté intime qui existe entre les puissances libératrices de la musicologie alternative et de l'ethnomusicologie, un domaine qui lutte encore pour sa reconnaissance au sein d'une académie musicale imbue de nostalgie myope et d'un désir de 
séparer les musiques d' «art » élitistes de toutes les autres formes d'invention musicale humaine. Cette tendence à mettre entre parenthèses tout ce qui peut être étiqueté comme «exotique», «traditionnel», «folklorique», «primitif », «commercial» ou «populaire» nous rappelle à quel point ces termes musicaux existent surtout parce qu'ils obéissent aux lois de l'élitisme et plus rarement parce qu'ils favorisent ou sont utilisés par ceux que l'on veut étiqueter à travers eux. Les musiques auxquelles ces termes réfèrent sont bannies de l'académie ou encore marginalisés par elle. Elles habitent une «réserve» qui peut, bien sûr, être visitée par les curieux, mais dont les clôtures proclament la corruption, la contamination, l'impureté de ses habitants. Bien que, pour plusieurs, le préfixe «ethno» du mot «ethnomusicologie» connote l'«ethnique» - rappelant immédiatement l'altérité exotique, non-occidentale de la réserve - , il faudrait nous souvenir que le vocable «ethno » n'est une désignation ni géographique ni chronologique et qu'il signale avant tout l'invention locale. L'ethnomusicologie est une discipline basée sur la reconnaissance qu'il existe de nombreuses musicologies locales et de nombreux discours sur la science et la pratique musicales; chaque «ethnomusicologie » correspond à un système d'invention musicale (théories, procédés de socialisation, pratiques, produits), système façonné par les modes de construction et de rationalisation d'une région donnée. Même si l'ethnomusicologie ne peut prétendre avoir privilégié la musique comme «un fait social total», son histoire est faite, d'une part, d'une quête rebelle pour établir l'autonomie des différentes musiques par opposition à une musicologie monolithique et, d'autre part, d'un désir d'insérer pleinement la musique dans le champ du social, et cela, en fondant l'étude du sonore sur l'ethnographique.

Il y a quelques années déjà, Charles Seeger insistait sur le fait que les historiens de la musique d' «art» de l'Europe occidentale, oeuvrant dans un esprit impérialiste et colonialiste, s'étaient approprié (ou, plus exactement, avaient «détourné») le mot «musicologie», instaurant, par la même occasion, un ordre hégémonique face auquel toutes les autres idéologies et pratiques musicales devenaient «comparatives» ou "ethno». La quête urgente des musicologies alternatives qui se fait sentir aujourd'hui me ramène aux propos de Seeger; elle indique la détermination avec laquelle les ethnomusicologues, les sociologues de la musique et le nombre grandissant de musicologues de la tradition européenne occidentale de la musique d' « art » cherchent maintenant un cadre critique commun, visant à décentraliser tous les discours privilégiés et à replacer la musique dans les débats au sujet des formations sociales. C'est dans ce contexte que s'insère le présent article; il se présente à la fois comme critique du discours musicologique dominant sur «l'analyse stylistique » et comme évocation d'une musicologie alternative du style ; le style imaginé et pratiqué par le peuple kaluli de la Nouvelle-Guinée papoue. Mais, avant même de présenter les Kalulis et le style kaluli, je veux 
examiner la notion de «style» dans d'autres rhétoriques vernaculaires et académiques.

\section{Le style comme «groove ${ }^{1}$}

Dans l'anglais vernaculaire, ce qu'on appelle un «groove» renvoie à un sens intuitif du style comme processus, à la perception d'un cycle en mouvement, à la révélation d'une forme ou d'un motif d'organisation, à un groupement récurrent d'éléments. De par leur stabilité et leur cohérence, ces traits formels s'identifient à leur contenu, mais ce qui les rend reconnaissables, c'est la façon dont ils modèlent un contenu spécifique à telle forme. Le "groove » et le style sont des essences distillées, des cristallisations dans le temps d'un ensemble d'expectatives. Nous conduisant justement vers la notion de "groove » musical, Leonard Meyer résume tout cela quand il dit : "Le style constitue l'univers du discours dans lequel s'organisent les significations musicales » $(1967: 7)$. Chaque " groove » culturellement construit constitue un tel univers, peu importe qu'une société se caractérise par des tendances à former un style monolithique ou des styles multiples; peu importe, également, que le style tende à la création du conflit et de la compétition ou à l'adoption et à la reproduction; peu importe, enfin, que la société en question situe plus généralement le style à l'intérieur d'autres modes culturels de désintégration, de fusion, de flux, d'acceptation et de rejet.

Instantanément perçu et accompagné, le plus souvent, de sensations de plaisir qui vont de l'excitation à la relaxation, le phénomène que l'on désigne par l'expression "getting into the groove » renvoie à la manière dont un auditeur socialisé s'attend à une certaine configuration dans un style donné; il renvoie aussi à la capacité immédiate de cet auditeur à distinguer et apprécier les subtilités malgré les régularités manifestes. «Getting into the groove» se rapporte aussi à la manière selon laquelle un exécutant expérimenté structure et maintient une cohérence perceptible. ${ }^{2}$ "Getting into the groove» se

1 Le terme " groove » a été en anglais parce que, référant à un très large éventail de significations, il n'a à ce titre, aucun équivalent en langue français; l'auteur l'utilisant beaucoup, et avec quantité de nuances subtiles, il aurait fallu le traduire chaque fois par un mot différent, ce qui aurait fait perdre le rôle de fil discursif qui lui est attribué tout au long de cet article. Soulignons toutefois que l'idée générale à laquelle renvoie le terme " groove » pourrait correspondre à " attraper l'esprit de la chose ». Signalons encore que S. Feld joue tellement sur le sens de « groove » qu'il le fait toujours figurer entre guillemets dans son texte; nous avons cru bon de conserver ces mêmes guillemets dans la présente traduction. (N.d.t.)

2 Keil (1966a) relie le " groove » aux processus subjectifs du balancement, du rythme vital et du sens métronomique dans le jazz. 
rapporte également à une participation active, à un état physique et émotif approprié, à un processus qui va de la réceptivité («hip to it») à l'engagement («getting down») et au fait d'être «dedans » (« into it»). Autrement dit, un "groove» est synonyme d'endroit confortable. Quand Aretha Franklin (1967) reprit à son compte la chanson de Sam Cooke intitulée « Good Times», elle remplaça la première ligne des paroles: «Come on baby and let the good times roll» ${ }^{3}$ par $~ « G e t$ in the groove and let the good times roll» ${ }^{4}$. Rappelons aussi comment James Brown (1966) l'exprime:

when your baby and you are tight

(quand ton amour et toi êtes proches l'un de l'autre)

and everything you say or do is mellow

(et tout ce que tu dis ou fais est doux)

she keeps everything alright

(elle s'assure que tout va bien)

and you know that you're the only fellow

(et tu sais que tu es le seul)

ain't that a groove!

(que c'est bon!)

Il va de soi ici que le "groove» musical dérive d'une référence originelle aux sillons creusés sur un disque, à ces impressions latérales observales sur sa surface. La musique se trouve mystérieusement «dans » ces renforcements physiques, collée au vinyle, et les auditeurs peuvent s'imaginer y séjourner et se fondre au sillon. Les styles sont gravés et enracinés dans les cultures de la même manière que les sillons sont gravés et enracinés dans les disques.

Tous les «grooves » ont des façons d'attirer sur eux l'attention des auditeurs, malgré leur subtilité; en outre, le sens intuitif de chaque personne à propos de l'esprit d'une musique correspond à sa reconnaissance du style en mouvement. Comme le dit Leonard Meyer: «Un style musical est un déploiement limité de relations et de mouvements mélodiques, rythmiques, harmoniques, de timbres, de textures et de formes. Quand ceux-ci sont intériorisés et deviennent des habitudes, les auditeurs (exécutants et compositeurs compris) savent percevoir et comprendre une composition d'un certain style en tant que réseau de relations imbriquées, ou expérimenter l'oeuvre comme un complexe de probabilités pressenties» (1967: 116) Pour Meyer, c'est à partir de ces relations qu'émergent «. . . les attentes les tendances - sur lesquelles se construit la signification musicale» (ibidem: 8; voir aussi Meyer 1956: 45-73).

3 «Viens, chéri, et que vienne le bon temps ».

4 « Mets-toi dans l'esprit et que vienne le bon temps».

5 C'est l'auteur qui souligne. 
Un raccourci linguistique comme le terme "groove» code la signification d'un sentiment à la fois ordonné et non spécifiable de quelque chose («les relations imbriquées» et «les probabilités pressenties» de Meyer) qui est soutenu d'une manière distincte, régulière, séduisante, et a pour conséquence d'entraîner l'auditeur. Des mots tels que «groove » disent que la perception du style est empiriquement réelle, mais qu'il s'agit aussi d'un phénomène général, vague et physique, fortement enraciné dans la sensation affective du temps et de l'espace. Comme le souligne Kenneth Burke : « une fois que l'on saisit la direction d'une forme, cela incite à la participation» (1969: 58, cité et discuté par Bauman 1977: 16). Leonard Meyer est du même avis lorsqu'il déclare : «Un style est appris, même par les compositeurs qui 'l'inventent' » (1967: 116) ${ }^{6}$. Il en va de même pour Clifford Geertz lorsqu'il écrit : «L'art et la capacité à le saisir sont faites dans le même atelier» (1983: 118).

\section{«Lift-up-over sounding $»^{7}$ : le «groove» kaluli}

Le présent article examine un cas spécifique et, à travers celui-ci, trois propositions ethnographiquement et théoriquement reliées :

1) La notion kalulie de dulugu ganalan, "lift-up-over sounding», est une affirmation de style, un "groove» propre à la Nouvelle-Guinée papoue. Le son «lift-up-over sounding» correspond au son kaluli, à l'expression locale d'une identité sociale qui s'articule à travers des essences acoustiques humaines.

2) Ce modèle sonore se manifeste directement dans le processus de création et la forme de la chanson kalulie, mais il se reflète dans d'autres modes d'expression et d'interaction des Kalulis; pour ces derniers, il est explicitement lié à l'écologie acoustique de la forêt tropicale environnante, ce qui témoigne chez eux de l'évolution parallèle des pensées esthétique et écologique.

3) Le processus selon lequel ce "groove » kaluli en vient à être senti comme complètement naturel correspond à une intensification expressive du style. C'est là où l'esthétique peut le mieux être comprise en tant qu'iconicité du style.

6 Les italiques et les guillemets sont de Meyer.

7 « Lift-up-over sounding » est intraduisible en français. En anglais même, l'expression est très difficilement compréhensible car elle se présente comme un amalgame insolite de termes qui ne correspond à rien de tangible; il s'agit, en fait, d'un néologisme créé par $S$. Feld dans le but de rester le plus près possible de la formulation des Kalulis, néologisme dont le sens se dévoile peu à peu à travers le discours de l'auteur. Comme le mot " groove », l'expression " lift-upover sounding ", toujours entre guillemets dans le texte original, se retrouve également entre guillemets dans la présente traduction. (N.d.t.) 
Les documents ethnographiques ici rassemblés concernent donc, avant tout, les Kalulis. Environ au nombre de mille deux cents, les Kalulis vivent dans la forêt tropicale du Grand plateau papou, sis dans la province Southern Highlands de la Nouvelle-Guinée papoue; j'ai vécu avec eux en 1976-77, en 1982, puis en $1984 .^{8}$ Sur plusieurs centaines de milles carrés de terre fertile située à une altitude d'environ deux mille pieds, ils chassent, pêchent et cultivent de grands jardins qui produisent des pommes de terre, des taros, des pandanus, des citrouilles, des bananes, et plusieurs autres fruits et légumes. Leur nourriture de base, le sago, provient de palmiers sauvages qui poussent dans des marécages peu profonds et dans des criques accrochées aux artères d'une large rivière qui coule au pied du Mont Bosavi, cône effondré d'un volcan atteignant huit mille pieds. Les Kalulis vivent dans une vingtaine des communautés distinctes; chacune de celles-ci comporte une maison-longue où résident quinze familles, soit environ soixante à quatre-vingt personnes. Mais ces dernières années, sous l'influence de pasteurs évangéliques et de fonctionnaires du gouvernement papous, il y a eu une vogue de maisons beaucoup plus petites, abritant une seule famille ou, tout au plus, deux ou trois.

Voilà une société égalitaire. Toutefois, avec l'intensification du contact avec l'extérieur des vingt-cinq dernières années, elle commence à ressentir l'impact du travail spécialisé, de la stratification et de la différentiation reconnues socialement. Manifestement, la vie quotidienne des Kalulis repose sur l'égalité, tant au plan social qu'au plan politique. Les gens chassent, font la cueillette, jardinent, travaillent pour produire l'essentiel, bref, ils pourvoient à leurs besoins, à ceux de leurs familles et amis dans un esprit de coopération totale: ils partagent la nourriture et le travail selon un mode d'organisation informelle où sont à l'honneur obligation et réciprocité. Bien que les différences entre hommes et femmes soient manifestement marquées, il existe toutefois chez eux peu de stratification consécutive à l'accumulation de biens et de récompenses ou, encore, au prestige. Cette dernière situation est en train de changer rapidement, certes, mais il existe encore - nos récentes expériences ethnographiques l'ont montré - un manque généralisé de déférence à l'endroit des personnes, des rôles, des catégories ou des groupes liés au pouvoir, à une position dominante ou à la propriété matérielle. Il y a des exceptions à cela, mais elles sont récentes; elles concernent les pasteurs, les Aid Post Orderlies, les travailleurs du gouvernement et les chargés de mission. Les différences liées au sexe ont aussi tendance à s'accentuer.

8 Pour une description ethnographique de base, voir E. L. Schieffelin (1976), Feld (1982) et B. B. Schieffelin (1990). 


\section{Style et métaphore}

Le terme kaluli dulugu ganalan, «lift-up-over sounding», est une métaphore spatio-acoustique; c'est, à la fois, une image visuelle traduite en forme sonore et une forme sonore traduite en image visuelle. On pourrait citer des exemples semblables provenant de vocabulaires musicaux de plusieurs langues, puisque l'imagerie spatio-visuelle constitue une source polysémique ou métaphorique de laquelle s'inspire la terminologie musicale. Bien sûr, considérée en elle-même, la figure verbale «lift-up-over sounding " laisse le champ libre à l'imagination. «. . . Dans le cas de la métaphore - écrit Owen Barfield - , . . c'est aussi le pur contenu de l'image, et non seulement sa référence, qui réjouit » $(1973: 70)^{9}$. L'essence de ce «pur contenu» est bon à penser; c'est une perception de forme et de fond à la fois imaginative et agréable. Aristote disait que la contemplation de la métaphore implique qu'il faille pénétrer dans la ressemblance, une pénétration que Paul Ricœur décrit comme un rapprochement de pensée, de sensation et de sentiment, «un modèle pour changer notre manière de regarder les choses, de percevoir le monde» (1978: 150). Et Roy Wagner d'ajouter : «Une métaphore est à la fois proposition et solution; elle se justifie d'elle-même» (1986: 11).

En dépit de sa densité, la métaphore «lift-up-over sounding » est, implicitement ou explicitement, discutée et réalisée par les Kalulis selon des manières équivalentes à ce qu'un étranger peut considérer comme étant quatre dimensions analytiques autonomes de la forme et du processus musical:

a) relations intrinsèques à un son instrumental et relatives aux propriétés acoustiques de cet instrument, et/ou relations entre ce son et d'autres sons environnants concomitants, produits intentionnellement ou non;

b) relations de coordination délibérée ou de simple coexistence de sons vocaux, chantés ou parlés;

c) relations issues de tout type de vocalisation avec les bruits d'accompagnement produits par des instruments ou des outils de travail, que les deux activités soient accomplies par les mêmes acteurs ou par des acteurs différents;

d) relations combinant n'importe lequel des points mentionnés ci-dessus à des sons simultanés de l'environnement (tonnerre, pluie, oiseaux, insectes, etc.).

Ce qui lie ces quatre dimensions est le «lift-up-over sounding»: celui-ci est toujours interactif et relationnel. Parce qu'ils attirent l'attention sur ces deux axes d'expérience que sont le spatial («lift-up-over») et le temporel («sounding »), le terme et le processus présupposent de manière explicite que chaque son spécifique intervienne toujours dans le champ de sons qui le

9 Les italiques sont dans l'original. 
précèdent et qui l'accompagnent. Ces qualités sont repérables à l'audition de tous les sons kalulis. ${ }^{10}$

Par exemple, dans le tambourinage kaluli, on peut distinguer plusieurs strates de «lift-up-over sounding»: la séparation du son fondamental et des harmoniques; les entrées décalées et les chevauchements des différents tambours; les relations imbriquées entre les sons provenant des sonnailles et du mouvement des costumes et les sons provenant des tambours. Toutes ces relations sont intensifiées et multipliées par la répartition spatiale des tambourineurs : ils dansent dans le corridor de la maison-longue, parfois à distance de cinquante pieds les uns des autres, parfois s'échangeant leur place, parfois encore, jumelés dans différents coins de la maison (Feld 1986). Autre exemple: le jeu de la guimbarde de bambou, dans lequel se combinent constamment, d'une part, les sons résonnant de la bouche et ceux provenant du pincement de l'instrument, et, d'autre part, le son fondamental et ses harmoniques; il y a ici deux strates interactives du «lift-up-over sounding».

Pour ce qui est du «lift-up-over sounding» vocal, il se réalise de diverses manières. Le chant solo peut prendre place sur accompagnement de sons de l'environnement (sons de cigales, par exemple), de sons produits par des outils de travail (machettes, haches, pilons à sago) ou, encore, des réverbérations des chutes d'eau sur un rocher. Le chant en duo, trio, quatuor ou groupe plus large (tous très communs) englobe aussi les accompagnements de hochet et les accompagnements instrumentaux, le sifflement, ainsi qu'une variété d'autres sons concomitants. De plus, chose très importante, tous ces types de vocalisation impliquent des entrées décalées et des chevauchements complexes, de manière à créer une continuité sonore permanente. Les sonnailles produisent également un son continu; même si la pulsation est résolument isométrique, les caractéristiques de l'enveloppe du son (attaque, entretien, extinction) font en sorte que le son de chaque pulsation empiète sur le début du son suivant. En outre, les sons produits par les costumes et les mouvements de danse sont en relation de «lift-up-over sounding» avec la vocalisation, et, dans les chants rituels exécutés pour provoquer les larmes, la présence du chant et des pleurs constitue une autre forme de «lift-up-over sounding ». Ces procédés se regroupent en six genres, dont quelques-uns sont entièrement rituels alors que les autres font partie de la vie de tous les jours.

Le «lift-up-over sounding» des Kalulis inclut des procédés que les musicologues occidentaux désignent par les termes de canon, hoquet, antiphonie et hétérophonie. Des termes descriptifs plus neutres - chevauchement, alternance et imbrication - ont été suggérés par Alan Lomax dans le but de distinguer les types de forme des processus dynamiques qui sont à l'œuvre

10 Tous les genres kalulis ont été enregistrés par Feld (1981, 1985 et 1987b). 
dans l'«organisation sociale du groupe vocal» (1976: 86, 177-180). ${ }^{11}$ Il semblerait toutefois que, dans certains cas, le terme "écho-polyphonie » soit plus approprié, étant donné la répétition par empiètement d'éléments mélodiques et textuels identiques ou similaires, éléments qui se dédoublent ainsi à distance de quelques secondes. Pour les Kalulis, tous ces phénomènes ressortissent au dulugu ganalan, au «lift-up-over sounding». Mais, en même temps, le terme dulugu ganalan ne circonscrit parfaitement aucune de ces données, et cela, parce que, pour les Kalulis, l'intervention de plusieurs voix ou instruments n'est pas indispensable. En effet, dans les cas de voix solo ou d'instrument solo, par exemple, il y a plusieurs densités, présences ou sources qui créent l'impression de strates, d'un son à plusieurs couches, d'une masse se dessinant dans le temps; les Kalulis disent que le dulugu ganalan est tout aussi présent dans ces exemples que dans ceux qui font intervenir plusieurs voix ou instruments.

\section{Particularités d'interprétation}

Abordons maintenant le problème sous un autre angle, c'est-à-dire à partir de l'unisson: ce type de son n'est jamais entendu dans les manifestations sonores des Kalulis, car il est l'antithèse du «lift-up-over sounding». Tous les sons «lift-up-over sounding» sont denses intimement mêlés et stratifiés; et s'il arrive parfois que les voix ou les types de sons coïncident momentanément, l'unisson qui en résulte est accidentel et éphémère, produit du pur hasard. L'essence du «lift-up-over sounding» se trouve dans les rapports entre parties qui sont à la fois en synchronie et en déphasage. En utilisant l'expression «en synchronie», je veux dire que l'impression globale en est une d'ensemble, qu'il y a coordination et cohésion du mouvement sonore et de l'expérience collective. Toutefois, les parties sont aussi «en déphasage", c'est-à-dire qu'à tout moment, elles se manifestent en des points différents et changeants du même cycle ou de la structure de phrase, chacun d'entre elles changeant continuellement de localisation par rapport à un unisson hypothétique.

En outre, dans le "lift-up-over sounding», le timbre (les fondements de la qualité sonore) et la texture (la sensation composite de la masse de sons en mouvement) prennent le dessus sur la syntaxe mélodico-rythmique; la performance et la forme se confondent, renforçant ainsi le principe d'interaction et des possibilités dialogiques du style. L'aspect multidimensionnel des exemples sonores est frappant. Le timbre et la texture ne sont pas de simples ornements; un noyau stylistique de «lift-up-over sounding " réside dans les fines nuances de densification de la texture, densification qui fait intervenir plusieurs plans: plans des attaques et des terminaisons de sons; des

11 Voir aussi Feld (1984: 391-2). 
extinctions de sons, des crescendos et decrescendos; des changements d'intensité, de profondeur et de présence; de la coloration et du grain de la voix; de l'interaction de sons intentionnels et de sons fortuits; des jeux d'accélération, d'extension et d'écourtement; de la désintégration et de la fusion des configurations sonores et des phrases, cela donnant lieu à ce que le compositeur Edgar Varèse appelait le chevauchement des couches de son à travers l'espace tonal. Une autre chose fait aussi partie du «lift-up-over sounding ", et à un titre très important: c'est la qualité que les acousticiens nomment "rustle time», à savoir, l'intervalle de temps moyen entre les déclics, les bruits ou les sons de hauteur indéterminée; ce phénomène est très audible dans les densitès palpitantes des hochets kalulis ainsi que dans les sons de l'environnement. ${ }^{12}$

S'il est vrai que des expressions comme "à la fois en synchronie et en déphasage» et "densification de la texture» peuvent, de prime abord, paraître maladroites, il n'y a aucune raison de croire que pareilles expressions sont moins généralisables que tout autre terme dont nous avons hérité ou qui est d'invention récente. Dans la tentative de clarifier cette question, Charles Keil (1987) propose un terme général: celui de «participatory discrepancies » («particularités d'interprétation»); il désigne par là ces dimensions de l'expérience musicale qui sont particulièrement irrécupérables, inanalysables ou négligées par les approches occidentales européennes de l'analyse de style, approches fortement orientées vers l'analyse syntaxique et hiérarchique de la mélodie, de l'harmonie et du rythme. Dans un article antérieur, Keil (1966a) abordait le même problème, confrontant la notion de «sentiment engendré" à celle de «signification incarnée » proposée par Meyer (1956). Il avait recours à la notion de «sentiment engendré » pour esquisser certaines propriétés formelles et expressives de la musique de jazz, propriétés rattachées à la performance, au processus, à l'instant et à l'improvisation. Il opposait ces propriétés aux dimensions identifiées par Meyer - drame, tension, inflexion architectoniques - comme porteuses de conséquences significatives dans l'expérience de la structure musicale par l'auditeur.

Sorte de version remaniée de cette critique, le concept de "particularités d'interprétation" proposé par Keil concerne deux niveaux spécifiques de tension créatrice dans la facture et l'écoute de la musique: le niveau du processus et le niveau de la texture. Le premier désigne des phénomènes comme «beat», «swing » ou "groove»; le second renvoie à des phénomènes tels que le timbre et la qualité du son. Selon Keil, c'est la «limite» mise en relief par les variations du rythme (processus) et d'intonation (la texture) qui génère la force vitale de la musique, et qui invite et garantit tout aussi bien

12 Cf. Schouten (1968) et, pour un panorama musical, Erickson (1975: 6, 71-2). 
la participation active. Chez les Kakulis, ce qui sert d'assise aux particularités d'interprétation au niveau du processus est le motif que j'ai appelé à la fois en synchronie et en déphasage, tandis que le fondement des particularités d'interprétation au niveau de la texture correspond à ce que j'ai nommé densification texturale. Ces phénomènes constituent les constantes dominantes du style interactif du dulugu ganalan.

\section{Travail, parole et «lift-up-over speaking»}

Autonomie coopératrice et collaboratrice: tel est le principe kaluli du style égalitaire et interactif. Que l'on imagine la notion de «synchronie anarchique», et l'on aura une idée de la façon dont travaillent les Kalulis. Ce que je veux dire, c'est que ce style interactif particulier renforce simultanément la participation sociale et l'autonomie du moi. Parlant de la manière vigoureuse et exubérante avec laquelle les Kalulis font généralement face aux situations, E. L. Schieffelin écrit: «Le sens de l'affirmation des Kalulis est lié à une compréhension implicite de l'autonomie personnelle ou de l'indépendance » (1976: 121); "les ordres proférés par les Kalulis donnent lieu à l'action, parce qu'ils sont excitants, bruyants et dramatiques» (ibidem: 129). Cette anti-concurrence - ou ce pro-mutualisme - se manifeste à travers l'égalité dans la participation et a souvent une qualité d'instantanéité, explosant dans ce qui est simultanément travail, jeu et performance. Chez les Kalulis, les démonstrations d'énergie, qui se traduisent souvent par de la flamboyance chez les hommes et de l'instance chez les femmes, «... se caractérisent par un degré élevé d'exubérance, de rassemblement de foule et de bruit» (ibidem: 154). ${ }^{13}$ "La présence de style dénote l'existence d'une communauté forte, d'une intense sociabilité qui a prise forme à travers le temps; elle signale aussi une affirmation du contrôle sur les sentiments collectifs, affirmation si puissante qu'elle incitera tout membre innovateur de la communauté à soummettre ses apports à cette construction collective et à nulle autre» (1985: 122).

Les sons produits par les Kalulis au travail - par exemple, des hommes coupant des arbres pour faire une emplacement de jardin ou des femmes en train de travailler le sago (Feld 1987b) —, indiquent comment le son, comme le travail, se fait essentiellement sans chef. «Lift-up-over» est l'image d'une action de groupe qui, tout en n'étant pas hiérarchisée, est synchronique, stratifiée et fluide. La dimension « jeu » reflète l'aspect expressément amusant du travail en tant que participation à une manifestation dramatique de coopération. Travailler ensemble, c'est "mener à bien" ("works out") et «travailler à » ("works through") un effet sonore collectif. "Mener à bien »

13 Voir aussi, pour une caractérisation plus approfondie de l'éthos affirmatif des Kalulis, E. L. Schieffelin (1985a). 
et «travailler à » correspondent aussi à la tension entre égalitarisme («faisons cela») et individualisme («je le ferai »). À vrai dire, «lift-up-over» est une image comparable à «s'écarter de » ou "s'élever au-dessus» de la foule, expressions signalant la même dialectique égalité/individualité. Le son réverbéré par le fait de travailler ensemble reproduit la qualité de «se joindre à » comme une modèle de sociabilité, de participation maximale et de distinction personnelle.

Telle est la situation non seulement pour le son musical ou environnemental, mais aussi pour le son verbal. La conversation des Kalulis est caractérisée par l'interaction: il s'agit d' "assembler les parlers », to kukan; cette dernière expression veut dire à peu près la même chose que "placer le bois » pour faire un feu. De la même manière que les morceaux de bois sont disposés de manière à être en contact, les voix s'imbriquent, alternent, se chevauchent et remplissent la moindre portion de l'espace-temps. Les conceptions normatives occidentales à propos d'interlocuteurs parlant à tour de rôle, de droits de parole, d'étiquette langagière - notions rationalisées dans la philosophie de la rhétorique et de l'analyse conversationnelle —, sont absentes des modes de conversation et de narration des Kalulis et n'ont, par conséquent, aucune pertinence analytique. Ce que l'on peut percevoir comme une «interruption» régulière n'est aucunement interruption; c'est plutôt l'aboutissement de la collaboration et de la co-création du dulugu salan, «lift-up-over speaking».

Jamais les Kalulis ne disputent les enfants parce qu'ils parlent tous en même temps; bien au contraire, les parents encouragent leurs petits à le faire, leur apprenant à «assembler les parlers » à toute conversation ambiante. C'est une autre manière de façonner la participation comme norme, comme obligation de sociabilité. Dans le développement de l'enfant, le cadre de socialisation du dulugu ganalan est manifeste jusque dans les interactions des jeunes qui apprennent à parler. Ceux-ci développent des contours vocaux prélexicaux stéréotypes qui se font écho, s'imbriquent ou alternent avec la parole qui les entoure. L'emploi de tels contours fait de l'enfant un partenaire et un collaborateur de la scène sociale ambiante, que le jeu et le mode d'apprentissage soient adressés à l'enfant ou non (B.B. Schieffelin 1983 et à paraître).

Ainsi, en ce qui a trait au parler, le dulugu ganalan ne correspond pas à une norme absolue; il s'agit plutôt d'une tendance susceptible de s'actualiser de manière plus ou moins accentuèe dans diffèrents types d'interactions et de situations verbales. Les performances verbales artistiques impliquent des attentes plus grandes et des réalisations intenses du dulugu ganalan, et, par là, accordent une grande importance à la participation de l'auditoire, lors même que la situation est définie par une forme monologique. En ce qui a trait aux interactions verbales quotidiennes, le caractère plus concentré des sujets et des événements (conversations animées, disputes, négociations, 
nouvelle information) favorise la tendance au dulugu ganalan dans les interactions où il est de rigueur de s'affirmer plutôt que de demander; au contraire, les stratégies de dulugu ganalan au sein de situations particulières comme celles de solliciter une faveur ou une complicité ou de rendre un autre tributaire de sa propre tristesse ne requièrent pas une dynamique de participation aussi manifeste.

\section{La forêt tropicale comme paysage sonore haute-fidélité}

Un matin à l'aube, alors que Gigio se promenait le long de ma maison, il m'aperçut assis sur le balcon arrière en train d'enregistrer les sons de la forêt. Il me regarda, sourit et s'exclama: «dulugu ganalan ena dadalega, wai, ni Bosabi nemo hoidake !, cle asulumeno.» ("J'entends le 'lift-upover sounding' là-bas, hé, mon Bosavi m'interpelle ! C'est ce que je pense.»)

Ce que nous entendions alors tous les deux était la forêt tropicale à l'aube (Feld 1987b), avec ses sons de bruines, de vents, de cours d'eau, d'insectes, d'oiseaux, de personnes, de porcs, de chiens, provenant d'une variété de lieux mais tous très audibles.

Qu'est-ce que Gigio voulait dire ? De toute évidence, que la forêt tropicale est un diapason qui donne des signaux bien connus pour marquer et coordonner l'espace, le temps et les saisons. «La perception de créatures par leur voix et leurs mouvements dans la forêt donne un sens particulier de présence et de dynamisme aux choses que nous ne voyons pas, à la vie invisible qui nous entoure ... Il est important de réaliser l'impression remarquable d'immédiateté des sons et des créatures entendues dans le calme pénétrant et l'immobilité de la forêt» (E. L. Schieffelin 1976: 96). On ne sait jamais combien de sources contribuent à la production de ces textures denses qui sont «à la fois en synchronie et en déphasage »; mais, peu importe leur nombre, la qualité sonore de la forêt est touffue et multidimensionnelle tout en conservant son unité, toujours globalement redondante sans jamais se répéter exactement.

Le compositeur R. Murray Schafer, chercheur reconnu dans le domaine des paysages sonores, qualifie ces derniers de «haute fidélité» lorsqu'ils contiennent un rapport signal-bruit favorable, c'est-à-dire quand l'échelle dynamique complète des sons présents peut être entendue clairement et distinctement, sans interférence, pollution, ou effets de masque produits par des sources de bruits étrangers (1981: 43). Schafer nomme « sons essentiels » ( «keynote sounds») les sons continus, fréquents, usuels, fondamentaux, qui confèrent un sens d'appartenance environnemental (ibidem: 8, 48). Ces notions sont tout à fait applicables au paysage de la forêt tropicale, où les sons renseignent sur la hauteur, la profondeur et la distance en forêt. Les Kalulis interprètent ces événements sonores omniprésents comme des unités 
de mesure de la réalité quotidienne, et eux-mêmes alternent constamment entre les états de réceptivité et de non-réceptivité de leur paysage sonore. Ainsi les Kalulis réadaptent-ils constamment leur centre d'attention, comme s'il s'agissait d'une lentille auditive qui modifierait ses angles de captation à mesure que les textures sonores de la forêt font bouger figure et fond et se métamorphosent à travers les cycles quotidiens et saisonniers.

Un facteur synesthésique peut aussi être à l'œuvre ici, qui porte à relier spontanément, quoique sur la base d'une convention culturelle, des particularités de son, de texture, d'espace et de mouvement. Dans la forêt tropicale, on peut facilement confondre hauteur et profondeur du son. L'absence de signaux visuels de profondeur se combine aux effets d'ambiguitté générés par les différentes densités de la végétation et aux sons omniprésents (comme le murmure de l'eau) pour faire en sorte que la profondeur du son soit souvent ressentie comme un mouvement vers le haut et vers l'extérieur, le son se dissipant à mesure qu'il s'éloigne. «Lift-up-over sounding» exprime cette sensation ambiguë de mouvement vers le haut et vers l'extérieur. La principale difficulté de mon adaptation à cet environnement fut d'apprendre à sentir et à distinguer la hauteur et la profondeur d'un son en l'absence de tout indice visuel. Même si j'étais conscient de l'évidence psychologique selon laquelle les humains sont plus aptes à localiser le son horizontalement que verticalement, et qu'ils ont souvent tendance à saisir les notes aiguës dans un registre plus élevé qu'elles ne le sont en réalité (Roffler et Butler 1968), je fus pendant des mois acoustiquement désorienté dans la forêt. Les Kalulis ont ri jusqu'à l'hystérie les premières fois qu'ils m'ont vu regarder en l'air à l'audition d'un son qui était grave, situé au-dessus ou à ras du sol. Et ils ont rapidement appris à me réorienter la main lorsque, par erreur, je dirigeais le microphone vers le haut en enregistrant un oiseau de la forêt.

La forêt est aussi le foyer mystique des «ane mama», («les reflets disparus»), esprits des Kalulis défunts. La présence de sons dénote la présence d'esprits à voix d'oiseaux qui se rérèlent ainsi par le son aux Kalulis. En ce sens, la réceptivité à la forêt peut susciter des sentiments puissants de nostalgie et de rêverie, même si les Kalulis n'attribuent aucun pouvoir surnaturel à la forêt en elle-même. La forêt procure également aux Kalulis un autre type de relation (sans doute moins cosmique, mais profondément satisfaisante): elle représente un lieu, un pays, un symbole d'identité. Pour les Kalulis, la forêt est agréable à écouter et il est agréable de chanter avec elle; les sons familiers donnent du plaisir et de l'inspiration. Le fait de devenir une partie de la forêt en chantant avec elle renforce en fin de compte les sentiments des Kalulis face aux conforts du chez-soi. Comme le dit Schafer: "L'homme fait écho au paysage sonore à travers la parole et la musique» (1981: 40). 
Écologie acoustique: les «dedans», les «dessous», les «reflets», la «fluidité», la «dureté»

Les habitants visibles qui peuplent la forêt tropicale du Bosavi sont toujours "lift-up-over sounding»; les Kalulis sont constamment à leur écoute, appréciant et interprétant ce qu'ils appellent les «dedans » (sa), les «dessous » (hego) et les «reflets» (mama) de ces pulsations omniprésentes. Ces expressions prolongent la notion de «lift-up-over sounding» et ont des implications sur l'engagement des Kalulis face à la forêt. Si dulugu ganalan constitue la métaphore générale employée par les Kalulis pour désigner une forme sonore naturelle, l'expression halaido domeki, «rendre dur», le la notion halaido de «dureté » qui s'ensuit évoquent sa matérialisation adéquate, sa réalisation canalisatrice d'émotion. "Dureté » est synonyme de force, d'obtention de cet état d'évocation, état chargé d'énergie ${ }^{14}$. Le pouvoir soutenu et continu de cet état de «dureté » correspond à son $\varepsilon b \varepsilon$ clan, c'est-àdire à sa "fluidité». Le "lift-up-over sounding» coule au-dedans de soi lorsqu'il y entre et y demeure, résidant dans la mémoire et la conscience de manière tout à fait inhabituelle. L'engagement - «getting into the groove» - correspond au fait de sentir le «dessous» («hego») et le «dedans» («sa») des configurations sonores qui «lift-up-over»; il correspond aussi à l'interprétation de leur «reflet» ou de leur «ombre» («mama») par association avec leurs forces et leurs possibilités. ${ }^{15}$ Il faut noter ici que le terme «reflet» ne fait pas référence à une stricte notion visuelle, comme lorsqu'on parle du reflet d'un miroir. Le sens est plutôt celui d'une « réverbération »: la projection d'une image ou l'ombre d'une essence qui est profondément intériorisée comme vibration, comme idée et comme sensation.

La participation - encore une fois, "getting into the groove — fait appel à un ensemble de gestes propres aux Kalulis: entendre le «life-up-over sounding », le sentir «durcir», le laisser «couler», le «retourner» pour trouver les «dedans» et les «dessous» qui y sont "réfléchis». Ici, non seulement les métaphores possèdent-elles leurs propres camarades de jeu linguistiques, mais ces dernières nous aident à comprendre la dimension spécifique évoquée par le tout: dimension qui touche à la fois le processus, la participation, l'interprétation, l'expérience, la sensation et l'intellect. Bien que le «lift-upover» semble faire appel à la verticalité, les Kalulis le comprennent plutôt dans le sens de propulsion, de courbe vers le haut et de débordement vers l'avant, comme un "groove» à la fois vertical et horizontal dans le temps. La complète cohérence spatio-acoustique du "lift-up-over sounding» s'échafaude à travers ses «dedans », ses « dessous », ses « reflets», sa « fluidité »

14 En cherchant à transposer cette notion en français, on trouverait des expressions comme « être sonné » ou " assommé », « être emporté ».

15 Pour plus de renseignements, cf. Feld (1984, 1986 et 1987). 
et sa «dureté». La poétique de l'esthétique se transforme en esthétique de la poétique. Le fait que ces métaphores nous ramènent à la totalité de ce qui peut être en relation et à la totalité de l'expérience rappelle le dynamisme des versions sociologiques et musicologiques du "getting into the groove» données par Alfred Schutz et Victor Zuckerkandl: «. . . partager le flux des expériences de l'autre dans le temps intérieur, vivant ainsi avec cet autre un présent commun constitue . . . la relation d'accord mutuel, l'expérience du 'nous'»(Schutz 1977: 115). Reprenant la notion de durée de Bergson, Zuckerkandl écrit: "l'audition d'un son musical est toujours comparable à une perception directe du temps. À l'instant où le son résonne, il nous tire dans le temps, nous ouvre le temps comme être percevants» (1956: 253).

\section{L'esthétique comme iconicité de style}

Des études ethnographiques récentes ont eu recours à des notions stylistiques telles que «homologie trans-modale» pour approcher la complexité de l'ethnoesthétique. Décrivant l'homologie comme «. . . relations logiques entre un ensemble de manifestations sociales et culturelles et les structures sous-jacentes qu'elles expriment», Adrienne Kaeppler (1978: 261) montre que, chez les Tongans, les formes visuelles, verbales, chorégraphiques et musicales sont construites comme une interaction à trois niveaux: fasi (mélodie, partie directrice, traits essentiels), laulalo (bourdon, marqueurs spatiaux) et teuteu (décoration, élaboration de détails). De manière similaire, l'analyse de Charles Keil (1979: 200-258) à propos des «cercles et angles » qui, chez les Tivs, servent de configurations matérielles et esthétiques, tient compte à la fois de matériaux et de modalités: toits, composés, terres, pots, calebasses décorées, scarification, gestuelle de danse, sculpture, percussion, narration, chant. Des analyses plus spécifiquement multi-sensorielles, synesthésiques, ont également été récemment présentées par Barbara Tedlock, qui retrace, chez les Zunis, l'opposition entre tso'ya (multi-coloré, chromatique, clair, brillant, tranchant, dynamique, varié) et attanni (le puissant, le tabou, l'obscur, le caché, l'hirsute, le vieux, le statique, le craintif) à travers la couleur, la décoration, le chant, le rituel et la cosmologie (1984 et 1986).

Les analyses de Judith et Alton Becker sur la coïncidence et la cyclicité dans les calendriers, le théâtre d'ombre et la musique de Java ${ }^{16}$ nous rapprochent davantage d'un « groove » puissant et fortement senti par le biais de la notion d'iconicité: «On peut appeler iconicité le non-arbitraire de toute métaphore. Les métaphores gagnent en force - et cessent même d'être prises pour des métaphores - à mesure qu'elles gagnent en iconicité ou en 'naturel' » (1981: 203). Ainsi, dans le cas des Kalulis, «le naturel» comme l'essence de la nature et de la nature humaine devient «le beau» - exactement et indis- 
cutablement comme cela devrait être. Lorsque ce que nous appelons des métaphores (comme «lift-up-over sounding») sont senties comme naturellement vraies, évidentes, complètes, absolues, elles deviennent alors iconiques, c'est-à-dire des symboles qui se tiennent en eux-mêmes (Wagner 1986), des images qui, tout en appartenant à des domaines ou des niveaux d'expérience différents, sont cependant ressenties comme synonymes. En termes sociologiques, comme le dirait Howard Becker: «. . . les gens n'expérimentent pas leur croyances esthétiques comme purement arbitraires ou conventionnelles; ils sentent qu'elles sont naturelles, correctes et morales » (1974: 773). Ces exemples d'études ethnoesthétiques rapellent la psychophysique à la manière allemande de la fin du XIXe siècle, laquelle est à l'origine de l'émergence, au XXe siècle, de l'anthropologie, de la linguistique et de l'ethnomusicologie: "Étant donné que le sensuel n'est perceptible que lorsqu'il a une forme, l'unité des sens est donnée dès le départ; de là, l'unité des arts ». ${ }^{17}$

Chez Pierce, la sémiotique de la similarité comprenait trois espèces d'icones: l'image, le diagramme et la métaphore (Pierce 1955). En reprenant ces termes, on peut dire que le «lift-up-over sounding» des Kalulis passe d'une métaphore de style (chez Pierce, la simulitude forme/contenu dans tous les signes) à une image d'identité (chez Pierce, le signe particulier rappelant directement son dénoté). Socialement parlant, le «lift-up-over sounding " passe d'une métaphore du "groove » kaluli à une icone que Meyer Schapiro appelait «... une manifestation de la culture comme un tout, le signe visible de son unité [qui] . . . réfléchit ou projette la 'forme intérieure' de la pensée et du sentiment collectifs» (1953: 287). Le «lift-up-over sounding» est une icone de ce que Sapir et Whorf, entre autres, ont appelé la nature intuitive d'une vision du monde ressentie, de ce que Paul Freidrich (1986) a décrit comme les dimensions émotionnellement satisfaisantes de l'indétermination poétique. C'est également à l'icone que Gregory Bateson fait appel quand, écrivant sur l'intégration psychique et sociale, il cite Buffon: «Le style est l'homme même" (1972: 130). Ce dont Voloshinov (ou Bakhtin?) propose une extension: "'Le style est l'homme'; mais nous pouvons dire: le style est, au moins, deux hommes ou, plus précisément, l'homme et son milieu social, incarné dans son représentant crédible, l'auditeur, qui participe activement au discours interne et externe du premier. $»^{18}$

Le sarcasme de Freud à l'effet que les analogies n'expliquent rien mais qu'elles ont au moins le mérite de nous faire sentir à l'aise trouve ici un écho. Certes, en devenant icones, les métaphores ont le réel mérite de nous faire sentir à l'aise; «sentir» est ici le mot critique puisque le lien entre jeu,

17 Von Hornbostel (1927: 89), cité par Merriam (1964: 99).

18 Cité par Todorov, qui traduit du russe (1984: 62). 
plaisir, cognition et émotion est ce qui donne de la valeur au «groove»: il s'agit non seulement d'entrer dans l'esprit de la chose, mais également d'en ressentir du plaisir. "Sentir, au sens émotif du terme», dit Ricœur, «c'est faire nôtre ce qui a été distancié par la pensée dans sa phase d'objectivation » (1978: 154).${ }^{19} \mathrm{Ce}$ « faire nôtre» est le plaisir extraordinaire et apparemment spontané, prévisible ou non, qui vient du «naturel » senti du tout, de la même façon que l'on se trouve dans/à travers la musique et que la musique se trouve dans/à travers nous. Plus la métaphore est iconique, plus sa cohérence est inconsciente, plus sa résonance est affective, plus son invocation est intuitive et plus son rayonnement est intense. "L'art est le globe brûlant du soleil de la signification» (Wagner 1986: 27).

\section{Synthèse: stéréotype et style}

Chaque élément du casse-tête stylistique se trouve dans le jeu des stéréotypes. Dans This Man and Music - méditation quasi autobiographique sur la musique en général et sur sa deuxième carrière de compositeur -, le romancier Anthony Burgess écrit: «La musique est considérée comme un langage international, bien qu'elle tende à un isolement flagrant. Qu'est-ce qui fait que la musique anglaise soit anglaise?» Un chef d'orchestre américain à qui je posais la question répondit, avec une certaine cruauté: «Trop de pièces d'orgue du genre 'voluntary' dans la Cathédrale de Lincoln, trop de couronnements à l'Abbaye de Westminster, trop d'envols de rossignols, trop de piétinements dans le foutu parc du village» (1982: 23). Ces stéréotypes du style national anglais (les cibles ici visées sont les excès de Holst, Elgar, Vaughn Williams) me rappellent un commentaire à propos de ce qui rend «kalulie» la musique des Kalulis, commentaire enregistré, en août 1982, au cours d'une discussion avec Keith Briggs, missionnaire évangélique résidant à Bosavi depuis 1971:

K.B.: Bon, je vais vous dire une chose que nous avons remarquée depuis des années; ces gens ne peuvent tout simplement pas chanter ensemble. Même lorsque nous comptons avant d'entonner un hymne, chacun suit son propre chemin après quelques mots.

S.F.: Est-ce parce qu'ils ne connaissent pas bien les hymnes ?

K.B.: Oh non ! Ils adorent les hymnes et sont tout le temps en train de les chanter autour de la mission . . . c'est vrai qu'ils apprennent les mots et les jeunes savent les harmoniser rapidement ... mais ils ne peuvent simplement pas chanter ensemble, même entre frères et sœurs . . . J'imagine qu'ils vont très bien respecter la mélodie . . . mais . . . jamais au même endroit au bon moment !

19 Les italiques figurent dans l'original. 
Les commentaires de Briggs ne sont ni aussi cruels ni aussi intelligents que ceux du chef américain, mais ils illustrent tout de même une compréhension de quelque chose d'essentiel à propos du style kaluli. Ici, la force du stéréotype agit différemment puisque Briggs interprète la force et la ténacité de la cohérence stylistique locale comme un signe d'inaptitude et d'infériorité musicales. Le choix de ses mots est révélateur: (1) «ils ne peuvent pas chanter ensemble», (2) «chacun . . . son propre chemin», (3) «jamais au même endroit au bon moment». Bien sûr, les Kalulis chantent vraiment ensemble, et avec un but commun; toutefois, l'unisson est aussi peu naturel à leur musique qu'aurait pu l'être l'improvisation microtonale libre dans l'Allemagne du XIXe siècle. Et, précisément, ce qui est le plus socialement interactif dans le «lift-up-over sounding» est compris par Briggs comme une tangente individualiste et anti-coopérative. Mais l'essence du dulugu ganalan, c'est d'être ensemble en se trouvant à différents endroits au (même) bon moment; de telle sorte que la direction prise par chaque personne nourrit et construit une interaction cumulative et coopérative.

En réalité, je soupçonne que ce qui dérange Briggs, c'est le fait que les Kalulis ne puissent être dirigés: c'est là, en fin de compte, une menace à son autorité et à son contrôle sur les chrétiens de sa localité. Il me vient à l'esprit les mots de Elias Canetti dans Crowd and Power: «Il n'y a pas d'expression plus évidente du pouvoir que la performance de direction d'un chef d'orchestre » (1963: 394). ${ }^{20}$ A vrai dire, les missionnaires ressemblent beaucoup à des chefs d'orchestre: à travers des petits mouvements de mains, ils ordonnent aux uns et captivent l'attention des autres, le message signifiant qu'il faut consentir à l'obéissance; et la confiance en soi est indispensable à la réussite de la performance. De plus, il est essentiel que les autres croient que ces gens vivent pour quelque chose de plus grand qu'eux-mêmes. Semblable à un dieu ou à un dieu substitut, le chef/missionnaire est omniscient, captant l'attention de tout le monde, connaissant et entendant chacun individuellement.

On voit ici le fonctionnement des inégalités de pouvoir, dont les dynamiques subtiles - particulièrement celles concernant la formation de faux consensus - ont été mises en place pour permettre la domination de classe ou «hégémonie». Bien que ce dernier terme ne s'applique pas ici dans le sens classique gramscien ou marxiste (Williams 1977: 108-114), il vaut la peine de rediscuter le ton idéologique qui émerge de la confrontation. Promulguer, comme le font les missionnaires, la naturalité du linéaire, du distinct, du séquentiel, du "un à la fois», de l'unisson, des rôles séparés du directeur

20 L'analyse de Canetti à propos du « chef d'orchestre " est citée et discutée en détail dans Keil (1979: 183-186). 
unique face au groupe d'exécutants, et de l'interaction sociale, faut preuve d'une idéologie de l'intrusion qui se proclame elle-même normale, supérieure et autorisée. Cela vise à légitimer et à rendre naturelle une opinion selon laquelle les Kalulis sont bruyants, indisciplinés, désorganisés, têtus, suivent «chacun leur propre chemin» et sont incapables de «faire les choses correctement ».

Les modes d'expression kalulis sont ainsi classés comme une simple " coutume », un terme courant en australien colonial (avec les mêmes nuances condescendantes et romantiques que le mot «folklore»), ainsi que dans la langue officielle de la Nouvelle-Guinée papoue - le tok pisin - où il se dit kastom. La "coutume» est ce qu'on demande aux Kalulis, auxquels on «permet» (en les payant parfois) d'exécuter, par exemple, un cérémonial de tambours d'une demi-heure le jour de la Fête de l'Indépendance de la Nouvelle-Guinée, à la piste d'atterrissage de la mission. En exigeant et en approuvant tacitement cette version officielle de l'expression, les gens de la mission signalent aux visiteurs dignitaires (d'autres agents de mission, des agents du gouvernement, des anthropologues) que les Kalulis «ont (encore) une culture». Mais, de manière sournoise, ils communiquent aussi que la "coutume » peut être accommodée, encadrée, contrôlée, allumée et éteinte, exhibée sur commande. Tel est le processus de la folklorisation hégémonique: les instances dominantes étrangères légitiment des démonstrations condensées, simplifiées ou accommodées; elles les invoquent, les promeuvent et les chérissent et tant que "coutume» officielle et authentique; mais, en même temps, elles comprennent mal, ignorent ou suppriment les forces créatrices réelles et les significations expressives que ces démonstrations animent dans la communauté.

Les Kalulis n'ont pas explicitement défié cette imposition, ils n'ont pas cherché à montrer de manière plus accentuée quelle était leur façon quotidienne de «faire les choses correctement»; ils n'ont pas cherché non plus à se démarquer outre mesure lors de ces performances commandées ou dans l'ensemble de leurs rapports avec la mission. Néanmoins, il est clair que leur style expressif n'est plus l'unique modèle naturel, cela annonçant pour l'avenir une série de confusions, de luttes, d'aliénations et de manifestations de résistance, d'autant plus que les sources missionnaires et gouvernementales ont déjà commencé à récompenser les performances par de l'argent et soutiennent que, pour être valables, les activités des Kalulis doivent se mouler dans d'autres cadres d'organisation.

\section{L'avenir: stabilité en tant que vitalité; «progrès» en tant qu'entropie}

Une chose est susceptible d'influencer significativement l'avenir d'une telle lutte en faveur du style bosavi: c'est la grande affinité du dulugu ganalan avec les nouvelles formes de musique populaire de l'ensemble de la 
Nouvelle-Guinée pan-papoue, que cette musique soit chantée en langue tok pisin, en hiri motu ou en quelque autre langue locale. En effet, le mélange de voix en synchronie et en déphasage, qui donne lieu à des polyphonies qui s'imbriquent et se chavauchent, s'allie à la métrique fortement marquée des accords de guitare ou de ukulélé pour constituer un canevas caractéristique de plusieurs musiques urbaines actuelles pour ensemble de cordes de la Nouvelle-Guinée papoue et de la région du Pacifique en général. Cette musique a de plus en plus fait son chemin vers Bosavi ces dix dernières années, soit par la radio (transmission à partir de Radio Southern Highlands, réseau provincial de la Papua New Guinea National Broadcasting Company), soit par les cassettes rapportées chez eux par les Kalulis travaillant ou étudiant à l'extérieur de Bosavi. Des groupes populaires de la NouvelleGuinée papoue tels «Panarama Strangers» ou «New Krymus Band» sont maintenant connus de la plupart des jeunes hommes kalulis, séduits par le son et la texture des voix et instruments qu'ils entendent; la compréhension des paroles (la plupart sont en d'autres langues que le tok pisin) est tout à fait secondaire, sinon sans importance.

En plus des appareils de radio-cassette - dont le nombre est passé de trois en 1976 à vingt-trois en $1984^{21}$ —, sont entrés au Bosavi, ces dernières années, cinq ou six ukulélés; les Kalulis s'en servent comme instruments de percussion de la même manière qu'ils jouent de leurs tambours et de leurs guimbardes de bambou. L'exécutant bouge les doigts de la main gauche comme pour produire des accords, mais en fait, ce sont des grappes de sons qui sont émises (consonantes ou dissonantes, et intentionnellement ou non) plutôt que des accords au sens conventionnel. Si les exécutants connaissent le mouvement de changement de positions des doigts, ils ne connaissent cependant aucun doigté d'accords et ne savent pas non plus accorder les quatre cordes de l'instrument. Le jeu de la main droite consiste en une succession de coups forts et isométriques, sans grande variation de volume ou de dynamique. Les Kalulis utilisent l'instrument à la manière d'un hochet rempli de graines, pour obtenir des effets de texture et comme support métrique, et non pour servir d'accompagnement d'accords à la mélodie d'un chant. L'effet recherché est la continuité d'un son dense et entretenu, sans trous, ni silences, ni vides. Les voix, qui «lift-up-over» l'une par rapport à l'autre, «lift-up-over» également par rapport au son du ukulélé, lequel suppose lui-même un «lift-up-over» de la chute de chaque son empiétant sur l'attaque du son suivant. Les résultat donne une grande densité de texture vocale/instrumentale, exactement comme dans tous les autres exemples de dulugu ganalan.

21 Le quart des propriétaires de ces appareils sont, non pas des Kalulis, mais des gens reliés aux secteurs de l'école, de la santé et de la mission. 
Les Kalulis n'accordent aucune valeur fétichiste à «l'innovation», au «progrès», au «développement» musical; ils ne pensent pas que le changement soit synonyme de vitalité et que le repos dénote la dégénérescence, notions que James Ackerman (1962) et Leonard Meyer (1967) ont soigneusement expliquées et défendues comme composantes de l'idéologie téléologique du «changement stylistique» dans les arts occidentaux. La fusion du dulugu ganalan avec le style populaire des ensembles de cordes qu'on trouve actuellement en Nouvelle-Guinée papoue constitue une excellente démonstration de la manière dont les Kalulis associent la vitalité à la stabilité. L'accomplissement de la nouveauté dans la musique populaire d'origine «kalulisée » - qui, rappelons-le, se répand dans toute la sphère du Pacifique - correspond, en fait, à une simplification (ou, à tout le moins, à une absence d'élaboration) des subtilités de timbre, de texture et de performance sur lesquels se fonde la construction du «lift-up-over sounding » dans les genres kalulis originaux. Mais la ressource stylistique de base est là pour permettre le développement de nouvelles nuances, à mesure que les Kalulis deviennent plus expérimentés et engagés dans l'usage, l'écoute et le partage de formes populaires néo-guinéennes papoues. Voilà précisément ce que disait Meyer en termes généraux: "Parce que le changement endostylistique n'implique pas une modification des prémisses d'un style, mais plutôt la réalisation de possibilités inhérentes à de telles prémisses, les changements endo-stylistiques ne sont pas nécessairement linéaires et cumulatifs. En conséquence, certaines œuvres venant tardivement dans la chronologie d'un style peuvent effectivement être moins complexes (plus redondantes) que celles qui les ont précédées » (1967: 120).

\section{«Voix bosavie»}

Une dernière histoire parlera clairement de la manière dont le son dulugu ganalan est également à l'œuvre au niveau affectif dans l'expression populaire de la Nouvelle-Guinée papoue. En 1976, Honowo apprenait la menuiserie à la Mendi Boys Vocational Training School; il avait été placé là par Keith Briggs (le missionnaire de Bosavi) en récompense de ses aptitudes linguistiques et techniques. Lorsque Honowo retourna à Bosavi pour les vacances de Noël (c'est à cette occasion que je le rencontrai pour la première fois), il apportait avec lui des bottes de travail, des lunettes de soleil et un ukulélé, signes de son intégration au monde citadin moderne de la Nouvelle-Guinée papoue. Un soir, Honowo vint chez moi chanter des chants en kaluli et en tok pisin avec Gigio, Seyaka et d'autres jeunes gens. Ils s'accompagnèrent au ukulélé (qu'ils jouaient à tour de rôle, chacun produisant un son identique, tel que décrit plus haut) et avec une version moderne du hochet kaluli appelé sologa, objet fait non pas d'une calebasse remplie de graines, mais de cailloux dans une boîte de conserve de poisson vide. 
Ce qu'ils chantaient là correspondait au vrai son kaluli « lift-up-over sounding »: les voix en synchronie et en déphasage (empiétant les unes sur les autres avec alternance de sauts à l'octave et de parties en falsetto), se mêlaient à la pulsation isométrique du hochet et du ukulélé, remplissant ainsi chaque portion de l'espace-temps sonore. À la fin d'un chant, Honowo se transforma spontanément en annonceur de radio, comme si le groupe s'était produit en direct sur les ondes de Radio Southern Highlands. Parlant en tok pisin, il identifia le chant qu'on venait d'entendre, et, comme ses camarades ricanaient, il présenta chaque chanteur par son nom et le lieu de sa maisonlongue, en le faisant précéder du mot Mista («Monsieur»). Il termina son intervention par un soupir nostalgique et en disant en anglais «ah yes, voice Bosavi» («ah oui, voix bosavie»).

En choisissant le mot anglais «voice» («voix»), Honowo ne faisait que reprendre l'habitude des annonceurs d'émissions radiophoniques provinciales de la Nouvelle-Guinée papoue pour qui le terme sert à identifier les styles locaux. ${ }^{22}$ Dans ce contexte, le mot "voix » réfère à la même idée que celle signalée par les termes "groove», «sound» et «beat». "Voice Bosavi» correspondrait alors à une manière néo-guinéenne contemporaine de paraphraser l'expression "sonorité kalulie», la manière dulugu ganalan. "Voice Bosavi » exprime ainsi la couleur locale d'une expression kalulie qui a trouvé sa «ples» (sa «place») et son identité propres à l'intérieur de ces mondes plus vastes constitués par la Province Southern Highlands et la Nouvelle-Guinée papoue.

\section{Une expérience personnelle de style}

Une autre ramification du dulugu ganalan mérite d'être expliquée: elle concerne la façon dont les Kalulis écoutèrent avec moi la musique de mon propre univers. L'expérience la plus mémorable que j'ai eue à cet effet se produisit un jour où je faisais jouer par plaisir une cassette de quelques pièces du Quintette de Miles Davis datant de la fin des années soixantes. En plus de Davis à la trompette, ce groupe était constitué de Wayne Shorter au saxophone ténor, Herbie Hancock au piano, Ron Carter à la basse, et Tony Williams à la batterie. Plusieurs des compositions jouées par le groupe étaient de Shorter, incluant «Nefertiti », une pièce tirée d'un album du même nom. Les oreilles Kalulies furent saisies. Cette mélodie de seize mesures confère une impression de division après huit mesures. Dans cet enregistrement, les seize mesures sont reprises treize fois par tout le groupe. La

22 Ils disent, en tok pisin, "bilong ples », « de cette place », i.e. de telle localité ou telle région; ou encore, «tok ples», " langue native », i.e. niveau de langue locale. Cf. Mihalic (1971: 157-8, 191). 
structure formelle est inhabituelle en ce qui a trait à la méthode d'improvisation des petits groupes de jazz. Normalement, ceux-ci commencent par jouer le thème («head») et improvisent ensuite des solos à partir du canevas harmonique et de son accompagnement rythmique («blowing» ou «playing changes»), avant de conclure avec une reprise en groupe du thème.

Dans «Nefertiti », le groupe au complet reprend plusieurs fois le thème. Mais cette interprétation implique de remarquables subtilités et différences dans chacun des treize refrains. De plus, les huit premières mesures de chaque refrain contrastent avec les huit dernières selon un principe de tensionrésolution. Plusieurs des subtilités soulignent les différences de texture entre la trompette et le saxophone ténor; plusieurs autres concernent les changements continuels de densité dans les parties revenant à la section rythmique (piano-contrebasse-batterie): ce que, dans le vocabulaire du jazz, on appelle "comping» (accompagnement du piano au moyen d'accords), «walking» (effet de marche, produit par la contrebasse) et «fills» (courts moments d'improvisation à la batterie, destinés à ornementer çà et là le jeu régulier en $4 / 4$ de la cymbale). Tout au long de la pièce, les changements de volume et d'équilibre entre les membres de la section des cuivres, entre les cuivres et les membres de la section rythmique, et à l'intérieur même de la section rythmique, ajoutent à l'effet de répétition nuancée et variée.

- La première fois, la mélodie est jouée uniquement par le saxophone ténor et la section rythmique.

- Il en va de même la fois suivante, sauf que, dans les huit dernières mesures, la trompette double le saxophone à l'unisson.

- Aux troisième, quatrième et cinquième reprises de la mélodie se développe un motif entraînant en $4 / 4$, avec les cuivres à l'unisson pendant que la section rythmique fait alterner des textures d'accompagnement plus ou moins denses; cette même section rythmique acquiert en outre de plus en plus de volume et ajoute davantage de fills, intensifiant ainsi, d'une reprise à l'autre, son rôle lors des deux dernières mesures de la phrase entière, mesures durant lesquelles aucun fragment mélodique n'est joué par les cuivres.

- A la sixième reprise, le volume diminue beaucoup et le piano se tait durant les huit premières mesures pour ne réapparaître qu'aux huit dernières.

- Le volume de la septième reprise est plus intense et la section rythmique y est plus active, occupant l'avant-plan dans les huit dernières mesures.

- La huitième reprise débute plus fort encore, puis diminue d'intensité pour redonner de l'importance à la contrebasse dans les huit dernières mesures.

- La neuvième reprise semble le moment culminant des solos de la section rythmique: on a d'abord un roulement d'accords plaqué au piano, qui joue en quatre contre six et en deux contre trois avec la contrebasse et la batterie; puis, dans les huit dernières mesures, un solo de batterie énergique fait entendre des motifs polyrythmiques analogues.

- A la dixième reprise, les cuivres jouent d'abord la mélodie selon une 
technique de réverbération («staggered overlapping echo»), revenant à l'unisson vers la fin.

- Tout au long de la onzième reprise, les cuivres font varier la technique d'écho et le jeu plus typique à l'unisson.

- La douzième reprise revient à la section rythmique uniquement: on y trouve de riches accords plaqués au piano, des effets de marche à la contrebasse («walking bass lines») ainsi que des fills polyrythmiques à la batterie se superposant à la pulsion de base («basic groove»).

- La treizième et dernière reprise ramène les cuivres à l'unisson, avec quelques légers effets d'écho. La pièce se termine sur un descrescendo de la section rythmique.

Les Kalulis ont été réceptifs à plusieurs dimensions de «Nefertiti» et ont appliqué leurs notions de "lift-up-over sounding" et de "dureté" pour commenter la structure formelle et les particularités d'interprétation de la pièce. Dulugu ganalan a servi de modèle pour discuter: (1) la relation entre la mélodie aux cuivres et son accompagnement simultané à la section rythmique; (2) les contrastes et les tensions de type figure-fond entre les moments d'ensemble et les moments solos, et tout spécialement les changements au piano et à la batterie; (3) le mouvement d'une phrase mélodique à l'unisson vers la phrase aux cuivres utilisant la réverbération; (4) le changement constant de sonorité et les contrastes insistants de volume, tant pour l'ensemble que pour différentes sections. De plus, la notion de «dureté» fut appliqueé à toute la structure, le point culminant étant localisé dans le passage couvrant la fin de la neuvième reprise - rythme mouvementé de la section rythmique jouant à forte intensité - et les huit premières mesures de la reprise suivante - phrase réverbérée des cuivres.

Il ne fait aucun doute que, pour bien apprécier cette interprétation particulière, il faille être attentif aux changements de texture et aux nuances de jeu du groupe qui, tout en intervenant simultanément, laisse à chaque musicien une certaine liberté d'expression. Du point de vue de l'auditeur de jazz expérimenté, le principe esthétique de la pièce repose sur un traitement ambigu de la distinction - classique en jazz - entre jeu d'ensemble et jeu solo. À la première écoute, «Nefertiti » ne contient aucun solo - du moins «aucun» selon le style d'improvisation classique du jazz - mais, en réalité, il y en a de part en part, les musiciens passant à leur gré de l'ensemble au solo. Je doute que ce soit là ce que les Kalulis aient entendu, et je ne veux aucunement suggérer qu'ils ont perçu «Nefertiti» de la même façon qu'un auditeur occidental, averti ou non de jazz, l'aurait fait. Toutefois, ils ne l'ont pas non plus simplement entendu comme quelque chose d'étranger. Bien que les Kalulis ne soient pas du tout familiers avec les styles mélodiques, harmoniques et rythmiques de la pièce et du genre, les éléments qu'ils ont perçus et nommé «lift-up-over sounding » sont spécifiquement des éléments interactifs et dynamiques, remplis de variations de densité et de séquences 
à la fois en synchronie et en déphasage. En réalité, ce qui faisait du sens pour les Kalulis dans «Nefertiti» ressemblait à leur style de «groove».

\section{Le «groove» kaluli}

La constitution du son «lift-up-over sounding» kaluli provient d'une dialectique à deux pôles: le pôle «son/environnement », d'une part, et le pôle "son/relations sociales», d'autre part. Le premier de ces pôles implique un processus d'adaptation. La conscience de l'environnement comme source d'inspiration musicale et la perception environnementale comme mode de connaissance musicale se situent dans le mouvement de va-et-vient entre la nature et le naturel, entre le senti et le sensuel. La musique de la nature devient la nature de la musique. L'autre pôle implique un processus d'intellectualisation. La conscience musicale en tant qu'identité sociale, c'està-dire l'idée interprétée à partir de la formation d'une idée, se situe dans le mouvement de va-et-vient entre le naturel et la nature humaine, entre le sensuel et le rationnel. Faire de la musique, c'est faire ce qui vient naturellement. La sensation simultanée d" "être dedans» et de "faire partie de» conduit à l'émergence du «lift-up-over sounding» en tant qu'image, une image synonyme, pour les Kalulis, du travail de groupe, de la fabrication de sons et de l'environnement sonore, chacun évoquant les mêmes configurations et les mêmes "grooves». Il se constitue une résonance externe vers l'interaction et le dialogue dans dulugu salan, dans ce «lift-up-over speaking ", vers l'intensification des textures aussi, de même que vers les relations - tant synchroniques que déphasées - entre costumes-danses-sons.

$\begin{array}{crc}\text { environnement: } & \text { son: } & \text { relations sociales:: } \\ \text { nature: } & \text { «naturel } »: & \text { nature humaine:: } \\ \text { senti : } & \text { sensuel: } & \text { sensibilité:: } \\ & & \text { rationalisation } \\ \text { adaptation } & \text { conscience<->identité } \\ \text { conscience<->inspiration } & \text { idée présentée<->idée réalisée } \\ \text { perception<->appéciation } & \\ \text { «dans» } & \text { «partie de» }\end{array}$

trope de dulugu ganalan

\section{Coda: style et "groove»"}

Comment le fait de considérer l'esthétique comme une représentation iconique du style détermine-t-il qu'il soit si séduisant de se sentir dans le «groove »? Le « groove» dulugu ganalan comble l'auditeur kaluli parce qu'il favorise un but ou un idéal social de participation maximale. Chaque effort 
de collaboration est à la fois un renvoi à soi, une habileté ou une compétence reconnues, une réalité tant révélatrice que gratifiante. En même temps, chaque individu est reconnu socialement comme agent coopérant, lié et plongé dans une myriade de relations humaines qui lui procurent continuellement la satisfaction du sentiment d'appartenance. Ce qui donne ce sentiment est l'intimité avec l'éthos de la culture, c'est-à-dire une façon d'être Kaluli chargée d'émotion qui, à la fois, maintient et provoque, agite et invite, stimule et console. Dulugu ganalan, c'est le jeu, le contrôle et le relâchement, la flexibilité et l'organisation. C'est aussi le poly- et le -phonique, ensemble mais toujours ouverts aux relations existantes, et en synchronie ou non. Il est bon de savoir comment se sentir bien. Keil (1987) et Gadamer (1986) ont suggéré de façon concise l'argument essentiel d'une réponse complexe: dulugu ganalan nous amène dans plusieurs directions de jeux connus, de performances, d'actions et de participations. En même temps, il s'agit d'explorer des formes et des expressions simultanément à leur création, d'amplifier la notion de «tuning-in» («ajustement») de Schutz et d' «instaurer - comme le dit Ricœur - des façons nouvelles de décrire le monde» (1978: 152).

Mais comment dulugu ganalan peut-il contribuer à la critique des tendances à objectiver les usages de la notion de «style» dans l'histoire de la musique occidentale ? Il est évident que le concept de style est souvent conçu comme une notion sans problème qui dénote une spécificité, une continuité, de la persistance, des frontières bien définies, une dynamique modérée et un potentiel de reproduction. Dans cette tradition, le «style» correspond, selon le système de classification le plus élémentaire, à ce qui est unique et spécifique à une pièce; il se définit également par la position occupée par un groupe de pièce dans un champ musical, champ dont elles découlent et vers lequel elles tendent. Le style est un assemblage de traits qui rend chaque pièce représentative d'un genre. En ce sens, le style peut dénoter les configurations sonores d'une période, d'une époque, d'un lieu ou d'un compositeur qui sont les plus redondantes, les plus facilement perçues et les plus homogènes structurellement; une réflexion fondamentale sur le lieu ou le temps qui passe est contrôlée, rejetée ou dépassée. Le style peut également être ce qui distingue une pièce faisant réellement preuve de créativité d'une autre qui n'y arrive pas. En outre, dans cette perspective, le style peut être considéré comme un phénomène aisément analysable, comme étant aisément compris, comme quelque chose qui se dédoit, purement et simplement, des notes d'une transcription ou d'une partition.

À titre d'exemple de ce «centrisme de la partition», le Guidelines for style analysis de LaRue se dit, dès la première phrase, "ancien et tributaire d'une lignée », se présentant par conséquent comme modèle sanctionné quant à son approche de la notion de style. Ce livre «... propose un cadre de référence pour comprendre la musique directement à partir des notes ...» (1970: vii). 
Un autre exemple, concernant cette fois la compréhension du style comme identité créatrice et distincte, se retrouve au début de $A$ Handbook of Style in Music, de Dickenson ${ }^{23}$ : "Le style reflète l'individualité d'une œuvre d'art, lui donne son identité. Cette identité résulte de la conjonction particulière d'éléments et des façons distinctes de mettre en relief ces éléments. Le style est ainsi la cristallisation des traits d'une ouvre, traits typiquement adaptés à une identité globale, c'est-à-dire à la personnalité créatrice de l'œuvre » $(1965: 3){ }^{24}$

Certains musicologues et théoriciens ont certes fait la critique de telles approches typologiques du style ou d'approches basées sur un ensemble de traits dérivés d'une partition, mais c'est indéniablement à Leonard Meyer (1979; 1967: 104-33) que l'on doit la réévaluation la plus importante et la plus rigoureuse des a priori en ce qui concerne le style musical notamment en ce qu'il a su mettre en lumière la confusion qui existe souvent entre classification et analyse stylistiques. Meyer (1979: 11) affirme que «. . . la connaissance du style n'est habituellement pas exprimée: il s'agit plutôt d'habitudes profondément ancrées (intériorisées) et utilisées de manière appropriée ». Il dit également: «C'est l'objectif des théoriciens de la musique et des analystes de style d'expliquer ce que le compositeur, l'interprète et l'auditeur connaissent implicitement». Et, plus loin: «Ce que le théoricien et l'analyste veulent donc connaître sont les contraintes de style en fonction desquelles les répétitions des configurations observées peuvent être apparentées entre elles et reliées à la connaissance des œuvres d'art » (ibidem: 13).

Meyer transforme malheureusement cette position en notion globale intériorisée et physiquement ressentie - de règles et de comportements, ce qui sous-entend qu'on ne peut étudier la nature des choix et des contraintes qu'à partir du comportement des musiciens tel qu'il apparaît à travers «les lois invariables d'un mode de connaissance humain" (ibidem: 14). Ce faisant, Meyer assujettit l'inévitable complexité sociale du style et néglige ainsi le rôle unique et fondamental de l'analyse culturelle dans l'interprétation de la richesse et de la profondeur du style en tant que représentation collective, ressource humaine et adaptation évolutive. De façon encore plus évidente, il sous-estime l'importance stylistique capitale des dimensions extra-musicales et socio-musicales pour expliciter ce qui fait spécifiquement partie du sonore-musical. Bien sûr, le problème est qu'une analyse complète du style met précisément en relief la division artificielle qui sépare le sonoremusical, l'extra-musical et le socio-musical, et qui, finalement, renvoie

23 On remarquera que la réflexion de Dickenson fait signe à plusieurs autres définitions du style.

24 Les italiques figurent dans l'original. 
l'objet d'analyse à son unité fondamentale. Il y a une cinquantaine d'années, M. M. Bakhtin a identifié un problème analogue dans l'analyse stylistique littéraire:

La démarcation entre style et langue, d'une part, et genre, d'autre part, a été largement responsable d'une situation où les connotations individuelles et chronologiquement limitées d'un style constituent les seuls sujets d'étude alors que sa dimension sociale fondamentale reste ignorée. La longue évolution historique des genres est occultée par les infimes vicissitudes des modifications stylistiques, lesquelles, à leur tour, sont liées à des individus ou à des courants artistiques. C'est pour cette raison que la stylistique, privée d'une authentique approche philosophique et sociologique, se retrouve dans une impasse de vétilles stylistiques; elle est incapable, au-delà des changements de nature individuelle et chronologiquement limités, de saisir les développements notoires et anonymes du discours artistique lui-même. Le plus souvent, la stylistique se définit elle-même comme une stylistique de l' «artisan privé», ignorante de la vie sociale du discours qui existe en dehors du studio de l'artiste, c'est-à-dire du discours tenu dans les lieux publics, les rues, les villes et les villages par les groupes sociaux, à travers les générations et les époques. La stylistique se préoccupe moins du discours vivant que du spécimen histologique que l'on en fait: un discours linguistique abstrait au service des talents créateurs individuels d'un artiste. Mais, lorsqu'elles sont coupées des contextes fondamentalement sociaux dans lesquels tout discours s'inscrit, pareilles connotations individuelles et tendancielles de style conduisent inévitablement à des formulations abstraites et sans vie; il devient impossible de les étudier en relation d'unité organique avec les composantes sémantiques d'une œuvre. (1981: 259)

En transposant l'exhortation de Bakhtin au domaine musical, on comprend encore mieux dulugu ganalan comme le complément d'une critique de caractère ethnomusicologique à propos de la réification historique des formes et des éléments fixes; dulugu ganalan se comprend également comme commentaire critique qui engage plus spécifiquement l'analyse musicoculturelle à situer la puissance du style dans l'imaginaire social et, comme le dit Schapiro, "[il] se comprend aussi comme l'incarnation ou la projection d'états émotifs et de modes de pensée communs à toute une culture» (1953: 305). D'un point de vue kaluli, le style (tel le dulugu ganalan) est plus qu'un reflet statistique de lieux, de temps ou de choix prédéterminés selon certaines contraintes. Ce sont les ressources humaines mêmes qui sont utilisées pour construire la réalité de la vie sociale à travers le son. Le style est en soi l'accomplissement ou la cristallisation de la participation individuelle et sociale. C'est la manière dont la performance et la participation dotent le sonore d'une forme humainement significative. Le style émerge comme un moyen par lequel un savoir nouvellement créé se développe à partir d'une expérience de jeu, de routine ou de participation habituelle. Le style influence l'expérience vécue en intériorisant et en rendant naturelles les pensées et les émotions ressenties. Outre sa capacité de conserver l'ordre 
musical du dulugu ganalan, le style sous-tend de manière créative l'expérience et la perpétue en accordant aux Kalulis le plaisir qu'un potentiel, des ressources, des compétences et des aspirations ont été exploités, tant à travers une écoute attentive que par une performance réelle.

Grâce au dulugu ganalan, les sensations, les sons, l'esprit de camaraderie typiques des Kalulis sont profondément intégrés aux plans cognitif et émotif, non seulement en tant qu'équivalents métaphoriques mais aussi en tant qu'entité iconique tangible. En ce sens, le style exprime l'essence de l'identité, et c'est pourquoi le dulugu ganalan kaluli agit comme médiateur entre la créativité individuelle et l'expérience collective; c'est aussi la raison pour laquelle les grooves/styles sont à la fois des univers de discours (Meyer), des éléments omniprésents et absolus (Schapiro) et, finalement, des manifestations de pouvoir (Keil).

\section{Remerciements}

Menée en 1976-1977, 1982 et 1984, la recherche chez les Kalulis a été généreusement subventionnée par les organismes suivants: the Institute of Papua New Guinea Studies, the National Endowment for the Arts, the National Science Foundation, the Wenner-Gren Foundation for Anthropological Research, the American Philosophical Society. Nous les remercions. Une version en anglais du présent article, accompagnée d'une cassette d'exemples musicaux kalulis du «lift-up-over sounding " a été publiée dans The Yearbook for Traditional Music (1988, volume 20).

(Traduction de Nicole Beaudry, Marcelle Guertin et Jocelyne Guilbault)

\section{RÉFÉRENCES}

ACKERMAN, J.

1962: «A Theory of style», Journal of Aesthetics, 20: 227-237.

ADAMS, R.N.

1975: Energy and Structure: A Theory of Social Power. Austin : University of Texas Press.

BAKHTIN, M.M.

1981: (original 1934-5): «Discourse in the Novel», The Dialogic Imagination: Four Essays. Michael Holquist édit., Austin: University of Texas Press, 259-422.

BARFIELD, 0.

1973: (1928): Poetic Diction : A Study in Meaning. Middletown: Wesleyan University Press. 
BAUMAN, R.

1977: Verbal Art as Performance. Prospect Heights, III : Waveland Press.

BECKER, A.

1979: «Text-building, Epistemology, and Aesthetics in Javenese Shadow Theatre ", The Imagination of Reality. A.L. Becker et Aram Yengoyan édit., Norwood: Ablex, 211-243.

BECKER, $\mathrm{H}$.

1974: «Art as Collective Action», American Sociological Review, 39 (6): 767-776.

BECKER, J.

1979: "Time and Tune in Java », The Imagination of Reality. A.L. Becker et Aram Yengoyan édit., Norwood: Ablex, 197-210.

BECKER, J. et A.

1981: «A Musical Icon: Power and Meaning in Javenese Gamelan Music », The Sign in Music and Literature. Wendy Steiner édit., Austin: University of Texas Press, 203-215.

\section{BROWN, J.}

1966: «Ain't that a Groove! », King Records.

BURGESS, A.

1982: This Man and Music. New York: Avon.

BURKE, K.

1969 (1950): A Rhetoric of Motives. Berkeley at Los Angeles: University of California Press.

CANETTI, E.

1963: Crowds and Power. New York: Viking Press.

DAVIS, M.

1967: «Nefertiti », Columbia Records.

DICKENSON, G.S.

1965: A Handbook of Style in Music. Poughkeepsie: Vassar College.

ERICKSON, R.

1975: Sound Structure in Music. Berkeley at Los Angeles: University of California Press.

FELD, $\mathrm{S}$.

1981: Music of the Kaluli, disque. Boroko: Institute of Papua New Guinea Studies (IPNGS 001)

1981: Sound and Sentiment: Birds Weeping, Poetics and Song in Kaluli Expression. Philadelphia: University of Pennsylvania Press. 
1984: «Sound Structure as Social Structure», Ethnomusicology, 28(3): 383409.

1985: The Kaluli of Papua Niugini: Weeping and Song, disque. Kassel: Bärenreiter (Musicaphon/Music of Oceania BM 30 SL 2702).

1986: "Sound as a Symbolic System: the Kaluli Drum», Explorations in Ethnomusicology in Honor of David P. McAllester. Charlotte Frisbie édit., Detroit Monographs in Musicology, No 9, Detroit: Information Coordinators, 147-158.

1987a: «Dialogic Editing: Interpreting how Kaluli read Sound and Sentiment », Cultural Anthropology, 2(2): 190-210.

1987b: Voices in the Forest, cassette stéréo. Austin, Texas : l'auteur (Wafe Sambo! 001).

FRANKLIN, A.

1967: «Good Times», «I Never Loved a Man the Way I Love You», Atlantic Records.

FREIDRICH, P.

1986: The Language Parallax. Austin: University of Texas Press.

GADAMER, H-G.

1986: "The Play of Art», The Relevance of the Beautiful and Other Essays. Robert Bernasconi édit., New York: Cambridge University Press, 123130.

GEERTZ, C.

1983 (1976): «Art as a Cultural System», Local Knowledge. New York:

Basic Books, 94-120.

HATTEN, R.S.

1982: Toward a Semiotic Model of Style in Music. Thèse de doctorat non publiée, School of Music, Indiana University.

HORNBOSTEL, E.M.V.

1927: «The Unity of Senses », Psyche, 7 (Avril): 83-89.

KAEPPLER, A.L.

1978: «Melody, Drone and Decoration: Underlying Structures and Surface Manifestation in Tongan Art and Society ", Art in Society. Michael Greenhalgh et Vincent Megaw édit., London: Dukckworth, 261-274.

KEIL, C.

1966a: «Motion and Feeling through Music», Journal of Aesthetics and Art Criticism, 24(3): 336-349.

1966b: Urban Blues. Chicago: University of Chicago Press.

1979: Tiv Song. Chicago: University of Chicago Press.

1985: «People's Music Comparatively: Style and Stereotype, Class and Hegemony », Dialectical Anthropology, 10: 119-130. 
1987: "Participatory Discrepancies and the Power of Music», Cultural Anthropology, 2(3): 257-283.

\section{LARUE, J.}

1970: Guidelines for Style Analysis. New York: W.W. Norton.

LOMAX, A.

1976: Cantometrics: An Approach of Music. Evanston: Northwestern University Press.

MEYER, L.B.

1956: Emotion and Meaning in Music. Chicago : University of Chicago Press. 1967: Music, the Art and Ideas. Chicago: University of Chicago Press.

1979: "Toward a Theory of Style», The Concept of Style. Berel Lang édit., Philadelphia: University of Pennsylvania Press, 3-44.

MIHALIC, F.S.V.D.

1971: The Jacaranda Dictionary and Grammar of Melanesian Pidgin. Queensland: Jacaranda Press.

PIERCE, C.S.

1955 (1893-1902): Philosophical Writings. J. Buchler édit., New York: Dover.

RICCEUR, P.

1978: «The Metaphorical Process as Cognition, Imagination and Feeling», On Metaphor. Sheldon Sacks édit., Chicago: University of Chicago Press, 141-157.

ROFFLER, S.K. ET BUTLER, R.A.

1968: «Localization of Tonal Stimuli in the Vertical Plane», Journal of the Acoustical Society of America, 43(6): 1260-1266.

SCHAFER, R.M.

1981 (1977): The Tuning of the World. Philadelphia : University of Pennsylvania Press.

SCHAPIRO, M.

1953: «Style», Anthropology Today. A.L. Kroeber édit., Chicago: University of Chicago Press, 287-312.

\section{SCHIEFFELIN, B.B.}

1983: «Talking like Birds: Sound Play in a Cultural Perspective», Acquiring Conversational Competence. E. Ochs et B.B. Schieffelin édit., London: Routledge and Kegan Paul, 177-184.

1990: The Give and Take of Everyday Life: Language Socialization of Kaluli Children. Cambridge: Cambridge University Press. 


\section{SCHIEFFELIN, E.L.}

1976: The Sorrow of the Lonely and the Burning of the Dancers. New York: St. Martin's Press.

1985a: "Anger, Grief, and Shame: Toward a Kaluli Ethnopsychology », Person, Self, and Experience. Geoffrey White et J. Kirkpatrick édit., Berkeley et Los Angeles: University of California Press, 168-182.

SCHOUTEN, J.F.

1968: "The Perception of Timbre», Reports of the Sixth International Congress on Acoustics, Tokyo, GP-6-2, 35-40 et 90.

SCHUTZ, A.

1977 (1951): «Making Music Together: a Study in Social Relationship », Symbolic Anthropology. J. Dolgin, D. Kemnitzer et D.M. Schneider édit., New York: Columbia University Press, 106-109.

SHEPHERD, J.

1977: «The 'Meaning' of Music», Whose Music ? A Sociology of Musical Languages. Par John Shepherd et al., New Brunswick: Transaction Books, 53-68.

\section{TEDLOCK, B.}

1984: «The Beautiful and the Dangerous: Zuni Ritual and Cosmology as an Aesthetic System », Conjunctions, 6: 246-265.

1986: "Crossing the Sensory Domains in Native American Aesthetics», Explorations in Ethnomusicology in Honor of David P. McAllester. Charlotte Frisbie édit., Detroit Monographs in Musicology, No 9, Detroit: Information Coordinators, 187-198.

TODOROV, T.

1984: Mikhail Bakhtin: The Dialogical Principle. Minneapolis: University of Minnesota Press.

WAGNER, $R$.

1986: Symbols that Stand for Themselves. Chicago : University of Chicago Press.

WILLIAMS, $\mathbf{R}$.

1977: Marxism and Literature. New York: Oxford University Press.

ZUCKERKANDL, V.

1956: Sound and Symbol: Music and the External World. Princeton: Princeton University Press. 